\title{
Potential Vorticity Diagnosis in the Quasigeostrophic and Nonlinear Balance Systems
}

\author{
John W. Nielsen-Gammon And David A. Gold* \\ Department of Atmospheric Sciences, Texas A\&M University, College Station, Texas
}

(Manuscript received 15 August 2006, in final form 12 April 2007)

\begin{abstract}
Quantitative diagnosis of low-Rossby-number flows using potential vorticity (PV) includes using elements of PV advection to deduce instantaneous tendencies of the balanced atmospheric state, most commonly the geopotential field. This technique, piecewise tendency diagnosis (PTD), is here applied with the prognostic balance equations (Bal-PTD) to obtain a quantitative dynamical diagnosis that in principle may be much more accurate than similar diagnoses using the quasigeostrophic (QG) equations.

When both are applied systematically to a case of rapid oceanic cyclogenesis, differences are found to arise owing to a variety of factors. The dominant factor is differences in the vertical influence of PV anomalies, which affects the partitioning between local and remote processes. QG overestimates the effect of lower-level PV, including surface potential temperature, in amplifying and controlling the motion of the upper-level system. Other differences are found, but overall the QG diagnosis gives results that are qualitatively similar to the nonlinear balance diagnosis. Quantitative accuracy requires the use of Bal-PTD.
\end{abstract}

\section{Introduction}

Various versions of potential vorticity (PV) are conserved in geophysical fluid flow under a wide range of physical regimes. For balanced flow, PV is often a valuable diagnostic quantity. Hoskins et al. (1985) illustrated a variety of diagnostic applications for midlatitude atmospheric motions, and subsequent research has further illustrated its utility. The various applications range in technical sophistication from inspection of isentropic maps of PV (Hoskins et al. 1985) to mathematical inversion of PV to recover aspects of the balanced atmospheric state (e.g., Davis and Emanuel 1991). While mathematical inversion is not required in many applications of $\mathrm{PV}$, the results from such inversion provide a quantitative benchmark with which the appropriateness of more qualitative aspects of PV thinking may be assessed.

PV inversion and diagnosis may also be performed with various governing equations. The quasigeo-

* Current affiliation: PPM Energy, Inc., Houston, Texas.

Corresponding author address: John W. Nielsen-Gammon, Dept. of Atmospheric Sciences, Texas A\&M University, 3150 TAMUS, College Station, TX 77843-3150.

E-mail:n-g@tamu.edu strophic $(\mathrm{QG})$ framework is the simplest: conventional QG diagnostic techniques are widely known, and inversion of PV in the QG framework is a linear operation. This linearity may be exploited through piecewise PV inversion, while inversion with more general equation sets requires arbitrary partitioning and assignation of the nonlinear terms (Davis 1992).

At least two significant disadvantages to the QG framework exist, however. First, the most common form of PV in the QG system is not truly an approximation to Ertel PV at all, but instead includes an additional term (Nielsen-Gammon and Gold 2008). Second, the QG system is formally valid over a limited range of parameter space, and in principle other approximate or truncated equation sets, such as nonlinear balance (NLB; Charney 1962), should perform better over a wider range of atmospheric conditions.

What is the nature of the inaccuracy of QG, particular for atmospheric flows for which QG is marginally valid? Aspects of this question have been addressed through life cycle simulations (e.g., Simmons and Hoskins 1976), diagnosis of vertical motion (e.g., Pauley and Nieman 1992; Vasilj and Smith 1997), and diagnosis of the vorticity equation (Grotjahn 1996). The specific answer to the question posed depends upon the context within which the question is answered. For example, Simmons and Hoskins (1976) found that QG and primitive equation baroclinic normal modes were similar, but 
noted that the neglect of vertical eddy heat transport in the QG system was a serious shortcoming. Rotunno and Bao (1996) examined an observed and simulated case of cyclogenesis and found that a QG model reproduced the essential features fairly well. We might expect, therefore, that QG diagnosis would give qualitatively correct answers overall. But both studies involve the diagnosis of a QG simulation, where internal selfconsistency is assured.

This paper addresses the issue of QG inaccuracy in the context of comprehensive observational diagnosis using PV. The analysis proceeds through a hierarchy of diagnostic techniques, from PV inversion to piecewise $\mathrm{PV}$ inversion to piecewise inversion of PV advection, formally known as piecewise tendency diagnosis (PTD). PTD (Nielsen-Gammon and Lefevre 1996; Evans and Black 2003) purports to identify and quantify specific dynamical processes and their roles in cyclone development. Of particular interest here is whether QG PTD properly assesses the relative importance of the leading dynamical processes. To explore this question a new version of PTD using the nonlinear balance equations (Bal-PTD) is implemented.

Section 2 of this paper reviews the principles of PTD and describes Bal-PTD. The data and methods of diagnosis are described in section 3. In section 4, differences between static inversions using QG and NLB are assessed. Section 5 compares PV advection and its inversion in the QG and NLB frameworks. The results are summarized in section 6 .

\section{Piecewise tendency diagnosis}

The QG system conserves, following the leadingorder horizontal wind, the pseudopotential vorticity (pseudo-PV) $q$ (Charney and Stern 1962):

$$
q=f+\nabla^{2} \psi^{(0)}+f_{0} \frac{\partial}{\partial p}\left(\frac{\theta^{\prime(0)}}{\hat{\theta}_{p}}\right)
$$

or

$$
q=f+\frac{1}{f_{0}} \nabla^{2} \Phi^{\prime(0)}-\frac{f_{0} p_{0}^{\kappa}}{R} \frac{\partial}{\partial p}\left(\frac{p^{1-\kappa} \partial \Phi^{\prime(0)} / \partial p}{\hat{\theta}_{p}}\right) .
$$

Here a caret represents a basic-state value constant in $x$, $y$, and $t$; a prime represents the departure from the basic-state value; the superscript 0 represents a leadingorder value; $f_{0}$ represents a reference value of the full Coriolis parameter $f$; and the subscript $p$ represents a derivative with respect to pressure. Other symbols have their usual meteorological meaning. In a traditional QG Rossby number expansion, the leading-order wind is geostrophic, so (1b) substitutes the Laplacian of the geopotential field for the Laplacian of the streamfunc- tion in (1a), and hydrostatic balance allows substitution of the vertical derivative of geopotential for the potential temperature.

Note that (1b) is an elliptic equation involving second derivatives of geopotential in both horizontal directions (the Laplacian) as well as the vertical direction. In symbolic form, (1b) may be written

$$
q=f+L\left(\Phi^{\prime(0)}\right)
$$

with $L$ being the three-dimensional Laplacian-like operator defined implicitly in (1b). Thus, given the height distribution, one may compute directly the pseudo-PV distribution. Alternatively, given the pseudo-PV distribution, one may solve for $\Phi^{\prime(0)}$ using standard techniques, such as successive overrelaxation, subject to constraints discussed in Hoskins et al. (1985). This process is known as inverting the Laplacian, or "inversion" for short. Written symbolically, the equation to be solved for the geopotential is

$$
\Phi^{\prime(0)}=L^{-1}(q-f) .
$$

Piecewise PV inversion (Davis 1992) divides the complete PV field into discrete anomalies, such as upperlevel and lower-level PV departures from a time mean, and determines the height field associated with each anomaly or set of anomalies using (3) or a higher-order counterpart. Such an exercise is relevant because ordinary baroclinic instability at its core involves the interaction between two counterpropagating Rossby waves at different levels (Hoskins et al. 1985). With piecewise $\mathrm{PV}$ inversion, the nature and influence of each Rossby wave can be isolated.

Ignoring diabatic and frictional processes, conservation of pseudo-PV is stated as

$$
\frac{\partial q}{\partial t}=-\mathbf{V}^{(0)} \cdot \nabla q
$$

This may be written using (2) as

$$
\frac{\partial L\left(\Phi^{\prime(0)}\right)}{\partial t}=-\mathbf{V}^{(0)} \cdot \nabla q
$$

or

$$
\frac{\partial\left(\Phi^{\prime(0)}\right)}{\partial t}=L^{-1}\left(-\mathbf{V}^{(0)} \cdot \nabla q\right) .
$$

Equations (5) and (6) are equivalent forms of the QG height tendency equation, written in terms of potential vorticity advection. While diabatic and frictional processes are neglected here, they may be incorporated as additional terms on the rhs of (5) or (6) (Evans and Black 2003).

PTD extends the technique of PV inversion by diagnosing height tendencies associated with PV advection. 
The advection is partitioned into advection of mean and anomalous upper-level PV by winds induced by mean, anomalous upper, and anomalous lower PV. The mean fields can be defined by any of several suitable means of temporal and spatial smoothing. The dynamical accuracy of PTD depends on the ability to accurately partition the wind and $\mathrm{PV}$, in addition to the accuracy of the relationship between pseudo-PV advection and height tendencies.

At first glance, the possible virtues of PTD are not apparent. Indeed, arbitrary subdivision of various forcing terms in the height tendency equation, like the arbitrary partitioning of vertical motion forcing into terms involving vorticity advection and temperature advection, is at best useless and at worst misleading unless each term involves a unique and relevant dynamical process. However, the partitioning of PTD does satisfy that constraint. The meaning of such a partitioning will be discussed in section 5. Here it is noted, following the earlier example, that the instantaneous effect of one Rossby wave packet on the motion and intensity of another may be directly quantified through this technique.

In the nonlinear balance system used by Davis and Emanuel (1991), the relationship between geopotential and streamfunction is nontrivial, so two coupled equa- tions must be solved to invert PV. Davis and Emanuel present the equations in spherical coordinates; for simplicity we repeat them here in Cartesian coordinates. We retain the Exner function $\pi$ as the vertical coordinate, and write the nonlinear balance PV as $Q$ to distinguish it from the pseudo-PV:

$$
\begin{aligned}
\nabla^{2} \Phi & =\nabla \cdot(f \nabla \psi)+J_{x, y}\left(\partial \psi / \partial x^{\prime} \partial \psi / \partial y\right) \\
Q & =\frac{g \kappa \pi}{p}\left[\left(f+\nabla^{2} \psi\right) \frac{\partial^{2} \Phi}{\partial \pi^{2}}-\frac{\partial^{2} \psi}{\partial x \partial \pi} \frac{\partial^{2} \Phi}{\partial x \partial \pi}-\frac{\partial^{2} \psi}{\partial y \partial \pi} \frac{\partial^{2} \Phi}{\partial y \partial \pi}\right] .
\end{aligned}
$$

The PV in the nonlinear balance system is not strictly the hydrostatic Ertel PV because the irrotational wind has been neglected in the last two (shear) terms of (8), but for simplicity we will refer to it as such.

The balanced counterpart to the quasigeostrophic PTD approach will here be called Bal-PTD. The version described here was first used by Gold (1996); a version utilizing only advection by the nondivergent wind was used by Bresky and Colucci (1996). Bal-PTD utilizes the prognostic balance equations introduced by Davis and Emanuel (1991), here again reproduced in Cartesian coordinates but with typographical errors in (9) corrected and diabatic and frictional processes neglected:

$$
\begin{aligned}
& \nabla^{2} \Phi_{t}=\boldsymbol{\nabla} \cdot\left(f \boldsymbol{\nabla} \psi_{t}\right)+2 J_{x, y}\left(\frac{\partial \psi_{t}}{\partial x}, \frac{\partial \psi}{\partial y}\right)+2 J_{x, y}\left(\frac{\partial \psi}{\partial x}, \frac{\partial \psi_{t}}{\partial y}\right) \\
& Q_{t}=\frac{g \kappa \pi}{p}\left[\begin{array}{c}
\left(f+\nabla^{2} \psi\right) \frac{\partial^{2} \Phi_{t}}{\partial \pi^{2}}+\nabla^{2} \psi_{t} \frac{\partial^{2} \Phi}{\partial \pi^{2}} \\
-\frac{\partial^{2} \psi}{\partial x \partial \pi} \frac{\partial^{2} \Phi_{t}}{\partial x \partial \pi}-\frac{\partial^{2} \psi_{t}}{\partial x \partial \pi} \frac{\partial^{2} \Phi}{\partial x \partial \pi}-\frac{\partial^{2} \psi}{\partial y \partial \pi} \frac{\partial^{2} \Phi_{t}}{\partial y \partial \pi}-\frac{\partial^{2} \psi_{t}}{\partial y \partial \pi} \frac{\partial^{2} \Phi}{\partial y \partial \pi}
\end{array}\right] \\
& Q_{t}=-\mathbf{v}_{h} \cdot \nabla Q-\dot{\pi} \frac{\partial Q}{\partial \pi} \\
& f\left(f+\nabla^{2} \psi\right) \frac{\partial}{\partial \pi}\left[\pi^{1-1 / \kappa} \frac{\partial}{\partial \pi}\left(\pi^{1 / \kappa-1} \bar{\pi}\right)\right]+\nabla^{2}\left(\frac{\partial^{2} \Phi}{\partial \pi^{2}} \bar{\pi}\right)-f \frac{\partial}{\partial \pi}\left(\frac{\partial^{2} \psi}{\partial y \partial \pi} \frac{\partial \bar{\pi}}{\partial y}+\frac{\partial^{2} \psi}{\partial x \partial \pi} \frac{\partial \bar{\pi}}{\partial x}\right)+\left(f \frac{\partial \nabla^{2} \psi}{\partial \pi} \frac{1 / \kappa-1}{\pi}-f \frac{\partial \nabla^{2} \psi}{\partial \pi^{2}}\right) \bar{\pi} \\
& =\nabla^{2}\left(\mathbf{v}_{h} \cdot \boldsymbol{\nabla} \theta\right)+f \frac{\partial}{\partial \pi}\left[\mathbf{v}_{h} \cdot \nabla\left(f+\nabla^{2} \psi\right)\right]-\nabla f \cdot \nabla\left(\frac{\partial^{2} \psi}{\partial \pi \partial t}\right)-\frac{\partial}{\partial \pi}(J) \\
& \nabla^{2} \chi+\pi^{1-1 / \kappa} \frac{\partial}{\partial \pi}\left(\pi^{1 / \kappa-1} \bar{\pi}\right)=0 .
\end{aligned}
$$

The first two equations are obtained as time derivatives of (7) and (8), (11) is the statement of conservation of
PV, (12) is the NLB omega equation, and (13) is the continuity equation. See Davis and Emanuel (1991) for 

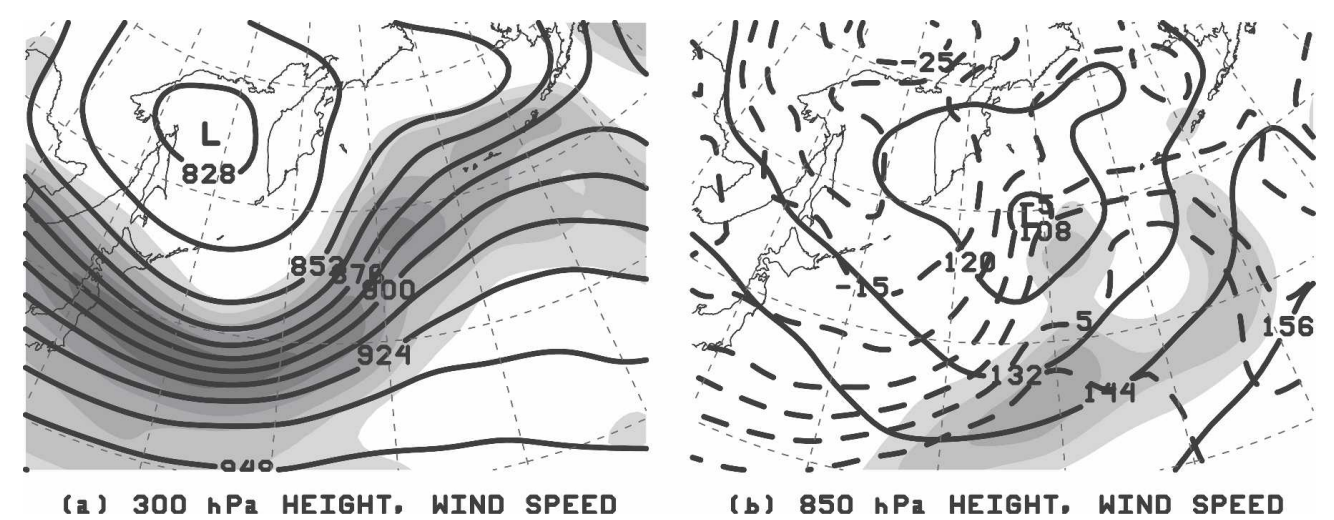

FIG. 1. (a) 300-hPa geopotential height (contours; dam) and wind speed (shaded above $30 \mathrm{~m} \mathrm{~s}^{-1}$ at 10 $\mathrm{m} \mathrm{s}^{-1}$ intervals). (b) 850-hPa geopotential height (solid contours; dam), wind speed (shaded above $20 \mathrm{~m} \mathrm{~s}^{-1}$ at $5 \mathrm{~m} \mathrm{~s}^{-1}$ intervals), and temperature (dashed contours; interval $4^{\circ} \mathrm{C}$ ). The time of this and all subsequent maps is 0000 UTC 1 Apr 1987. Adapted from Nielsen-Gammon and Gold (2008).

details. The $J$ in (12) represents the same Jacobian terms as appear in (9). The horizontal wind $\mathbf{v}_{h}$ in (11) and (12) is the sum of the nondivergent wind (obtained from $\psi$ ) and the irrotational wind (obtained from the velocity potential $\chi$ ). The prognostic balance equations consist of five equations in the following five unknowns: streamfunction tendency, height tendency, PV tendency, vertical velocity, and velocity potential.

The balance equation set obtained through truncation of the equations governing the streamfunction and velocity potential do not have the property of PV conservation (McWilliams and Gent 1980). In the Davis and Emanuel prognostic balance equations, PV conservation is enforced by replacing the equation for the tendency of vorticity with (11).

For Bal-PTD purposes, the PV tendency associated with advection of a certain portion of the PV field by a certain portion of the balanced velocity field is used to solve the remaining four equations and recover the associated piecewise height tendency. While piecewise inversion of PV using the nonlinear balance (NLB) system involves nonlinear terms that must be partitioned arbitrarily (see Davis 1992), Davis and Emanuel (1991) pointed out that the prognostic equations of NLB are entirely linear in the five unknowns, so piecewise tendency inversion presents no such difficulty.

\section{Data and methods}

The data source is the National Centers for Environmental Prediction-National Center for Atmospheric Research (NCEP-NCAR) reanalysis (Kalnay et al. 1996) in spherical harmonic, sigma-coordinate form. The 6-hourly gridded analyses were sampled onto a $2.5^{\circ}$ latitude/longitude grid ( $1.875^{\circ}$ for QG calculations) and interpolated to a 50-hPa vertical interval. The grid spacing corresponding to the effective resolution of the spherical harmonics was $1.875^{\circ}$, but not all the NLB inversions converged at full resolution.

The case chosen for study is a rapidly deepening extratropical cyclone over the North Pacific. This event was chosen to present a stringent, but not severe, test for QG dynamics. The event is synoptic in spatial scale and there are no topographic gravity waves of importance, but the rapid intensification of the storm may stress the validity of the QG system.

The 300-hPa and $850-\mathrm{hPa}$ heights and winds at 0000 UTC 1 April 1987 are shown in Fig. 1 (adapted from Nielsen-Gammon and Gold 2008). A strong short wave is embedded within a long wave trough over the western North Pacific at $300 \mathrm{hPa}$. Downstream of the short wave, an $850-\mathrm{hPa}$ low is present along a baroclinic zone. The low is in the process of intensifying rapidly (not shown). The quarter wavelength of the developing system is $1000 \mathrm{~km}$ and the characteristic wind speed in the upper troposphere is $50 \mathrm{~m} \mathrm{~s}^{-1}$, giving a Rossby number of 0.5 .

The time-mean fields for the diagnostic calculations are computed as a 7-day average centered at 0000 UTC 1 April 1987. The time of the diagnostic calculations presented here corresponds to the rapidly deepening phase of the cyclone. Other times produce qualitatively similar results, with varying apparent effects from nonlinearity and latent heat release as the cyclone evolves.

The QG calculations are performed similarly to Nielsen-Gammon and Lefevre (1996) except that the horizontal and vertical grid spacings were as listed above. The pseudo-PV is partitioned into upper-level and lower-level PV with the dividing line at the 550-hPa level. The top and bottom boundary conditions were $\theta$ 
at $75 \mathrm{hPa}$ and $975 \mathrm{hPa}$, respectively; when inverting lower-level PV the top $\theta$ anomaly was set to zero, and the bottom was set to zero for upper-level PV. Inversion was performed over the entire globe, eliminating the need for lateral boundary conditions. Although the inversion will not be locally valid where $f$ is significantly different from $f_{0}$, such as in the tropics or the Southern Hemisphere, the inversion operation is everywhere well behaved.

G. Lawson (1998, personal communication) found two errors in the description of the inversion algorithm by Nielsen-Gammon and Lefevre (1996): the term on the third line of their (A.3) has the wrong sign and the term on the fourth line of (A.3) should involve the sum of the reciprocals of the sigmas rather than the reciprocal of the sum of the sigmas. These errors were not present in the numerical algorithm itself.

The NLB inversions were performed over the same vertical domain as the $\mathrm{QG}$ inversions but over a limited horizontal domain bounded by $20^{\circ}-80^{\circ} \mathrm{N}, 50^{\circ} \mathrm{E}-$ $132.5^{\circ} \mathrm{W}$. Some minor lateral boundary effects are apparent, but a larger horizontal domain introduced convergence issues because of small or negative values of PV in the tropics.

The prognostic balance equations have been utilized previously by, for example, Davis and Emanuel (1991) and Davis et al. (1996), to obtain the balanced vertical velocities, diabatic generation, and full PV advection. Here we solve them to determine balanced height tendencies. The prognostic inversion procedure begins with a computation of $\mathrm{PV}$ advection by the nondivergent wind. This may be the complete PV advection or just a piece, for example advection of time-mean PV by the nondivergent wind associated with upper-level PV anomalies. The advection field is inverted for the balanced height and streamfunction tendencies. The NLB omega equation is then solved, and the irrotational wind computed using the continuity equation. With the new vertical motion and irrotational winds, a new PV advection field is computed. The process is repeated until the maximum change in the height tendencies from iteration to iteration is less than $0.1 \mathrm{~m}(12 \mathrm{~h})^{-1}$. The effects of moisture, particularly latent heat release within the cyclone updrafts, are not included in the NLB diagnosis so as to facilitate comparison with the QG diagnosis, which is also dry.

\section{Differences in static PV inversion}

The elements of piecewise diagnosis that might lead to discrepancies between the QG and NLB systems are 1) differences in the PV distribution, 2) differences in static PV inversion, 3) differences in PV advection, and
4) differences in the inversion of PV advection. This section considers the first two elements.

Element 1 was considered by Nielsen-Gammon and Gold (2008), who compared pseudo-PV with a scaled Ertel PV. The direct comparison is shown here in Fig. 2. The scaled Ertel PV $\breve{Q}$ is defined as

$$
\breve{Q} \equiv \frac{Q}{-g \hat{\theta}_{p}}=\frac{\left(f+\zeta_{\theta}\right)}{\hat{\theta}_{p}} \frac{\partial \theta}{\partial p} .
$$

This scaling makes Ertel PV anomalies directly comparable to pseudo-PV anomalies, although the scaled Ertel PV is not conserved.

The neglect of ageostrophic vorticity causes a redistribution of PV, making the pseudo-PV too positive within cyclonically curved flow and too negative along the perimeter of the cyclonically curved flow. The neglect of nonlinear terms tends to make the pseudo-PV too weak within Ertel PV anomalies and too strong beyond the horizontal and vertical edges of Ertel PV anomalies. The neglect of the tilting term causes the pseudo-PV to be too positive within frontal zones. Nielsen-Gammon and Gold (2008) noted that the differences arising due to neglect of ageostrophic vorticity are not actually inherent in the QG approximation, but arise in the conventional framework because the geopotential height is usually taken as exact when QG diagnosis is applied to the real atmosphere. Alternative applications of QG diagnosis based on the observed nondivergent wind, for example, do not require the neglect of ageostrophic vorticity.

Differences in static inversion can arise through differences in the PV itself such as those discussed above as well as differences in the inversion operator and differences in the partitioning. The effects of the inversion operator were considered by Bishop and Thorpe (1994) and Thorpe and Bishop (1995). Thorpe and Bishop (1995) explicitly considered the induced response to a point PV anomaly located some distance beneath a tropopause-like jump in stratification and found that the response would decay much more rapidly with height in the approximate Ertel PV case than in the QG case.

Situations beyond this simple case seem not to have been explored, but there are reasons to suppose that the solutions for more realistic distributions of PV are quite a bit messier. The vertical response in $\mathrm{QG}$ versus NLB, for example, might be different in the case of a wavelike series of PV anomalies. The familiar streamfunction response to a single point vortex of strength $Q_{0 i}$ located at $\left(x_{i}, y_{i}, 0\right)$ is (Bishop and Thorpe 1994)

$\psi^{(0)}=-\frac{1}{4 \pi} \frac{Q_{0 i}}{\left[\left(x-x_{i}\right)^{2}+\left(y-y_{i}\right)^{2}+z^{2} N^{2} / f_{0}^{2}\right]^{1 / 2}}$. 


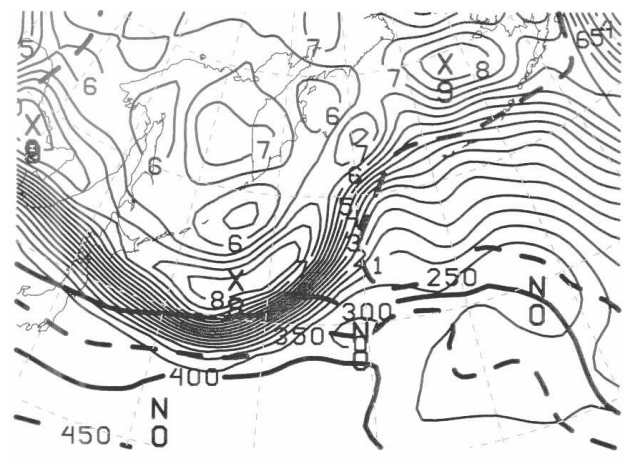

(a) 325 K ERTEL PV (PVU), P ( $\mathrm{PPa})$

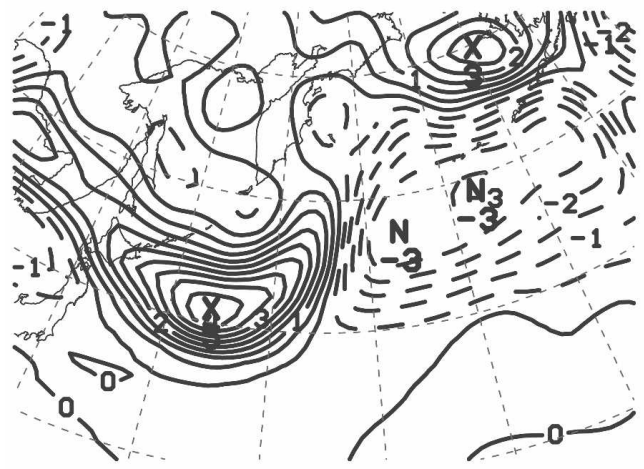

(c) $300 \mathrm{hPa}$ SCALED ERTEL PV ANOMALY



(b) $300 \mathrm{hPa}$ SCALED ERTEL PV

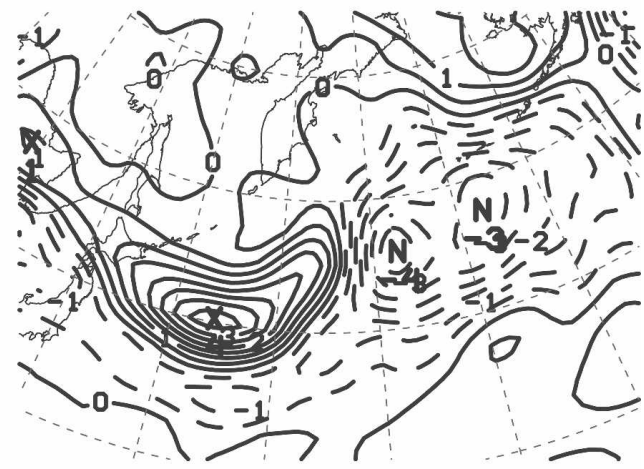

(d) $300 \mathrm{hPa}$ PSEUDO-PV ANOMALY

FIG. 2. Total and anomalous potential vorticity over the North Pacific. (a) Ertel PV (PVU; thin solid) and pressure (hPa; thick alternating solid/dashed) on the $325-\mathrm{K}$ isentropic surface. (b) Ertel $\mathrm{PV}\left(\times 10^{-4} \mathrm{~s}^{-1}\right)$ on the 300-hPa isobaric surface, scaled according to (14). (c) As in (b) but for anomalous scaled Ertel PV (time-mean PV removed). (d) Anomalous pseudo-PV $\left(\times 10^{-4} \mathrm{~s}^{-1}\right)$ on the $300-\mathrm{hPa}$ isobaric surface, computed from the analyzed geopotential heights. Adapted from Nielsen-Gammon and Gold (2008).

Directly over the point vortex, the vertical decay goes as $1 / z$; there is no inherent vertical or horizontal scale to the response. However, a more general case is $(2 M+1)$ alternating-sign point vortices arranged in a line symmetrically about the origin, each separated by a distance $L_{Q}$ from its neighbors, with the outermost vortex pair having half the amplitude of the other vortices so that the sum of the vorticity is zero. This situation is an idealization of a Rossby wave train along a jet stream or a surface frontal zone. In that circumstance, directly over the origin,

$$
\begin{aligned}
\psi^{(0)}= & -\frac{Q_{0}}{4 \pi}\left\{\frac{1}{z N / f_{0}}+\sum_{m=1}^{M-1}\left[\frac{(-1)^{m} 2}{\left(m^{2} L_{Q}^{2}+z^{2} N^{2} / f_{0}^{2}\right)^{1 / 2}}\right]\right. \\
& \left.+\frac{(-1)^{M}}{\left(M^{2} L_{Q}^{2}+z^{2} N^{2} / f_{0}^{2}\right)^{1 / 2}}\right\} .
\end{aligned}
$$

The response for various values of $L_{Q}$ within a vertical column centered over the middle vortex, in the case of $M=6$, expressed as a fraction of the single point vortex solution (15), is shown in Fig. 3. The single point vortex solution is the limit of (16) as $L_{Q}$ approaches infinity. For finite $L_{Q}$, the response is much smaller than the point vortex case when $z$ becomes larger than the Rossby height $H_{R}$ :

$$
z>H_{R} \equiv \frac{f_{0} L_{Q}}{N \pi} .
$$

At $z=H_{R}$, the response is 0.6 times the response to an isolated point vortex. For $L_{Q}$ less than about $4000 \mathrm{~km}$, the Rossby height is reached at less than tropopause depth, resulting in significant departures from the point vortex solution within the troposphere.

The differences in the inversion operators also may be used to deduce differences in the QG and NLB PV distributions. Consider an isolated positive surface $\theta$ anomaly overlaid by horizontally uniform Ertel PV in the troposphere and stratosphere. The atmosphere will relax to some particular steady-state height and wind distribution. The PV computed using NLB will be very similar to Ertel PV; assume they are the same. Then the anomalous heights computed from inverting the full 


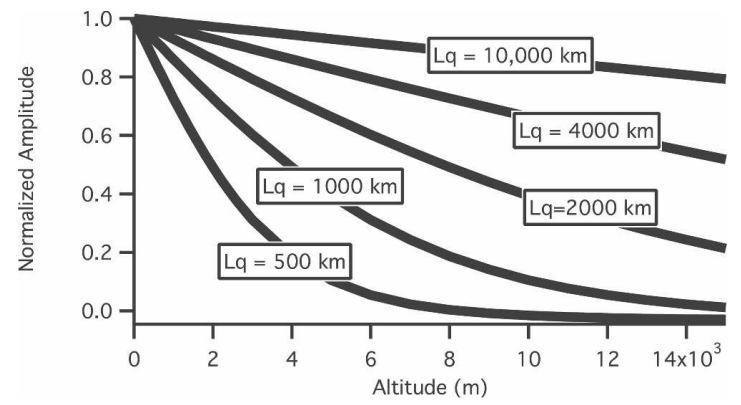

FIG. 3. Amplitude of the balanced height response over a string of point vortices separated by distance $L_{Q}$. The amplitude is normalized by the response at the same altitude to an isolated point vortex. See text for details.

three-dimensional PV distribution will equal the anomalous heights computed from inverting the surface $\theta$ alone, and both must agree with the actual anomalous heights. Likewise, with QG, the inversion of the full pseudo-PV distribution, including boundary $\theta$, must recover the full anomalous heights, but the pseudo-PV is not guaranteed to be uniform within the interior.

For the piecewise inversion of the surface $\theta$ anomaly, Thorpe and Bishop (1995) show that the QG response with horizontally uniform pseudo-PV will decay more slowly with height than the NLB response with horizontally uniform PV. Therefore, there must be some nonzero pseudo-PV anomaly in the interior of the fluid which, when inverted together with the lower boundary using QG, recovers the original balanced height field. Since the QG response to surface $\theta$ decays too slowly, or is "too strong" aloft, the interior pseudo-PV must be negative so as to partly shield the surface $\theta$ from the interior and produce the correct overall vertical decay rate. Therefore, the pseudo-PV cannot in general be horizontally uniform even when PV is horizontally uniform.

Differences in the partitioning arise because, unlike the QG and prognostic balance inversions, the static NLB inversion is nonlinear. The partitioning of the nonlinear terms is arbitrary, and Davis (1992) considers several alternative partitioning schemes. We follow the partitioning approach used by Davis and Emanuel (1991).

We now turn to the actual event. Figure 4 shows the anomalous heights obtained from inverting upper-level and lower-level PV (and boundary $\theta$ ) within both the QG (Figs. 4a-d) and NLB (Figs. 4e-h) frameworks. The panels extend beyond the limited domain of the NLB inversions so that the impact of the homogeneous lateral boundary conditions is apparent. Heights are plotted at both 300 and $850 \mathrm{hPa}$. Not surprisingly, the heights induced at the same level as the anomalies
(Figs. 4a,d,e,h) have higher amplitudes than the heights induced at more distant levels (Figs. 4b,c,f,g). Such same-layer heights are similar between the QG and NLB inversions. The most notable differences are with the amplitude of the 300-hPa upper-level-induced positive height anomaly over the central Pacific (Figs. 4a,e: $\mathrm{QG}$ is stronger) and with the structure of $850 \mathrm{-hPa}$ anomalies over Tibet (Figs. $4 \mathrm{~d}$,h: caused partly by below-ground interpolations that are inherently balanced in the QG framework but not necessarily so in the NLB framework).

Also not surprisingly, the more distant levels (Figs. $4 \mathrm{~b}, \mathrm{c}, \mathrm{f}, \mathrm{g})$ possess smoother fields. The smaller-scale details in the height field decay more rapidly with height because they have a smaller Rossby height (Fig. 3). It is seen, as expected from Thorpe and Bishop (1995), that the $300-\mathrm{hPa}$ heights induced by low-level pseudo-PV (Fig. 4c) are of higher amplitude than the corresponding NLB heights (Fig. 4g). The larger negative height anomaly over the central Pacific (Fig. 4c) is compensated for by the stronger positive height anomaly noted in the previous paragraph (Fig. 4a) to yield a total height field that agrees closely with the total NLB height field (not shown).

The low-level height induced by upper-level PV is more intense in the NLB inversion (Fig. 4f) than in the QG inversion (Fig. 4b). This stronger downward penetration in NLB is opposite to the weaker upward penetration in NLB seen in Fig. 4g (compared to Fig. 4c). A comparison of tropopause-level anomalies was not explicitly made by Thorpe and Bishop (1995), so we do not know if this result is general. In this particular case, upper-level PV anomalies are more strongly felt at the surface in the NLB framework.

\section{Differences in piecewise potential vorticity advection and inversion}

Aspects of the dynamics of this cyclogenesis event will now be diagnosed using PTD. We will employ a top-down approach in comparing the performance of the two PTD techniques. We will first assess the overall accuracy of each technique by examining the total diagnosed and analyzed height tendencies. Then we will look at the height tendencies associated with specific mechanisms to gain insight into how QG and NLB diagnoses differ in their portrayal of the most significant processes. The key to the differences in the height tendency fields can be found by examining the associated PV advection fields, which, in turn, comprise winds and heights attributed to various $\mathrm{PV}$ anomalies through piecewise inversion.

To limit the number of variables, we will confine our attention to the heights associated with the upper-level 


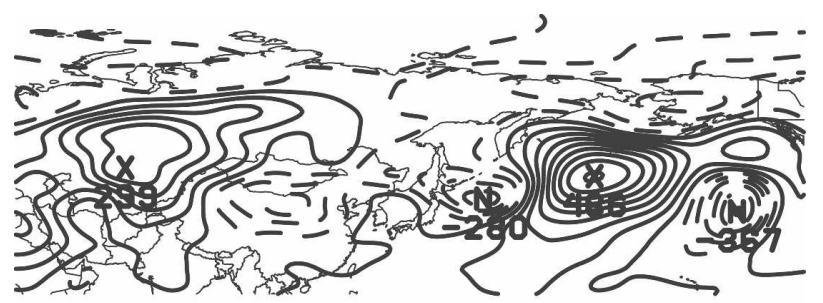

(a) $300 \mathrm{hPa}$ HEIGHT FROM UPPER PSEUDO

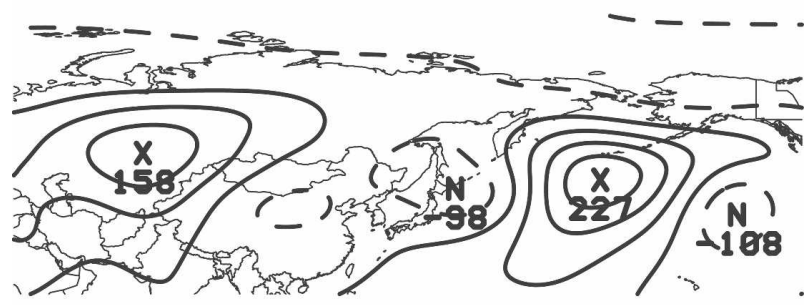

(b) $850 \mathrm{hPa}$ HEIGHT FROM UPPER PSEUDO

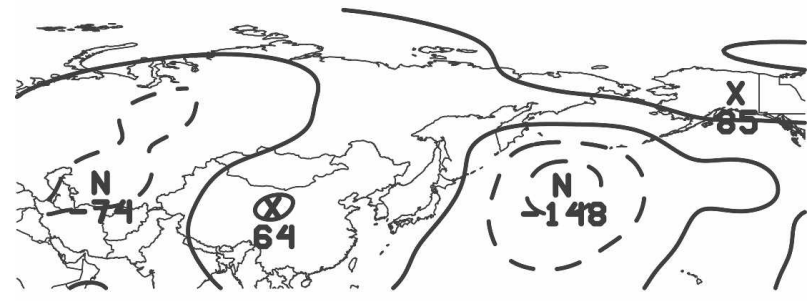

(c) $300 \mathrm{hPa}$ HEIGHT FROM LOWER PSEUDO

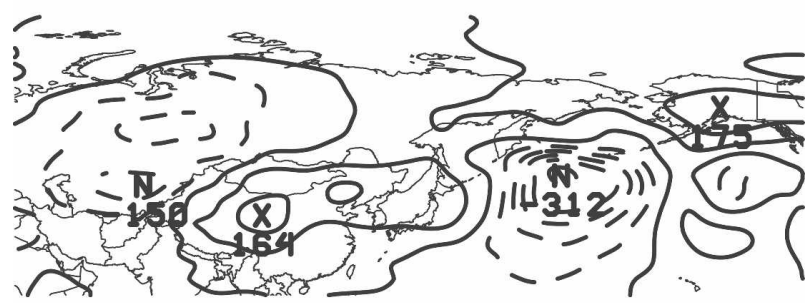

(d) $850 \mathrm{hPa}$ HEIGHT FROM LOWER PSEUDO

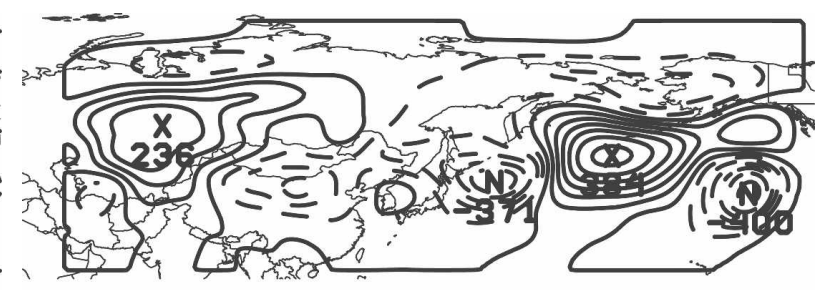

(e) $300 \mathrm{hPa}$ HEIGHT FROM UPPER ERTEL

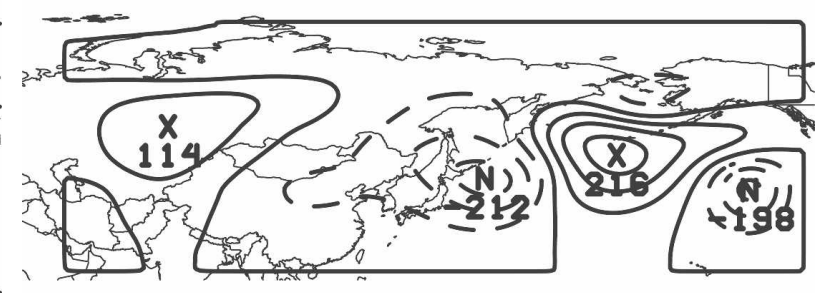

(f) $850 \mathrm{hPa}$ HEIGHT FROM UPPER ERTEL

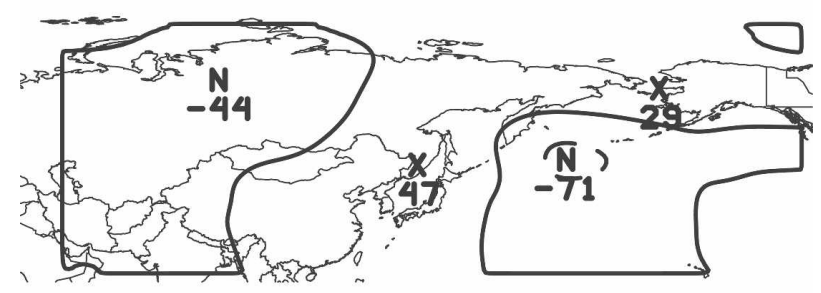

(g) $300 \mathrm{hPa}$ HEIGHT FROM LOWER ERTEL

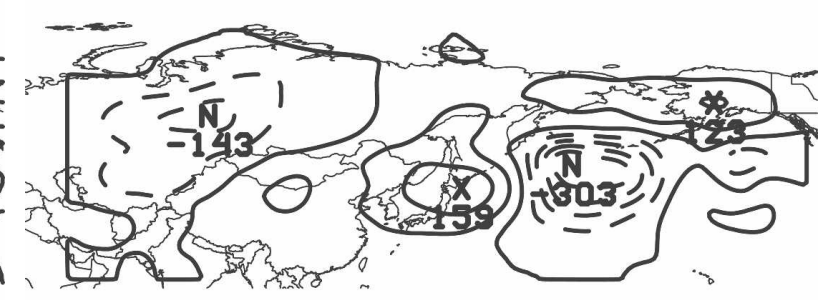

(h) $850 \mathrm{hPa}$ HEIGHT FROM LOWER ERTEL

FIG. 4. Heights induced by anomalous upper- and lower-level potential vorticity (and boundary potential temperature, 0000 UTC 1 Apr 1987). (a)-(d) Quasigeostrophic inversions. (e)-(h) Nonlinear balance inversions. (a), (e) Heights at 300 hPa induced by upperlevel PV. (b), (f) Heights at $850 \mathrm{hPa}$ induced by upper-level PV. (c), (g) Heights at $300 \mathrm{hPa}$ induced by lower-level PV. (d), (h) Heights at $850 \mathrm{hPa}$ induced by lower-level PV. Contour interval is $50 \mathrm{~m}$; negative contours are dashed.

anomalous PV only. Thus, we will be diagnosing changes in the upper-tropospheric PV anomalies and the heights and winds associated with those anomalies.

Even though PV advection is the key forcing, we will not display the mathematical quantity "advection" but, instead, will overlay wind vectors and contours. Doing so makes the larger scales of advection easier to perceive and masks the smaller scales, corresponding to their relative importance for height tendencies. Doing so also preserves the information regarding the strength and orientation of both winds and gradients, making it possible to determine how sensitive the advection would be to small changes in either. Advection magnitudes can be readily inferred from the (fractional) number of contours crossed by the wind vectors.
Figure 5 shows the diagnosed and analyzed 12-h tendencies of the heights induced by the upper-level PV. The diagnosed tendencies are computed for 0000 UTC 1 April 1987, while the analyzed tendencies are a centered difference between 1800 UTC 31 March 1987 and 0600 UTC 1 April 1987. The heights associated with upper-level PV are computed as for Figs. 4a and 4e by inverting the upper-level PV; the centered difference of the static inversions at two different times constitutes the analyzed tendency. The centered difference smoothes the tendency field; similar smoothing of the diagnosed tendencies using multiple times (not shown) produces a closer match with the centered differences. Just as the upper-level heights at $300 \mathrm{hPa}$ were similar (Figs. 4a,e) between the QG and NLB calculations, so 


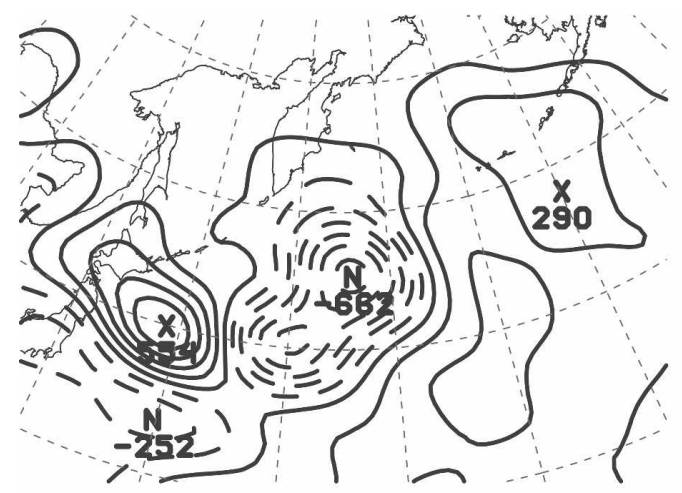

(a) DIAGNOSED OG HEIGHT TEND

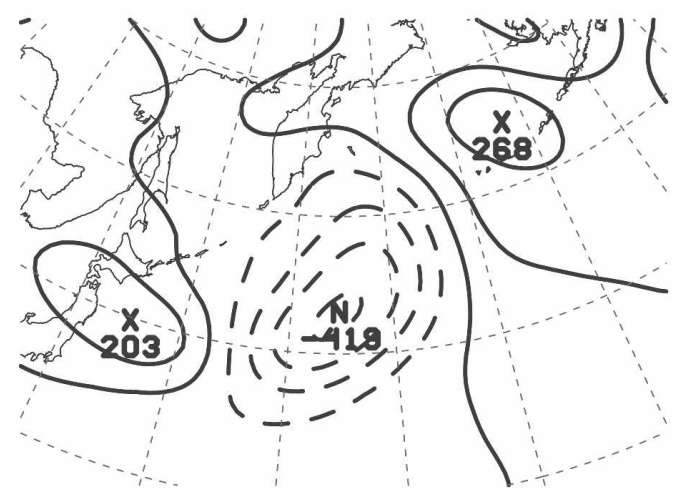

(c) ANALYZED QG HEIGHT TEND

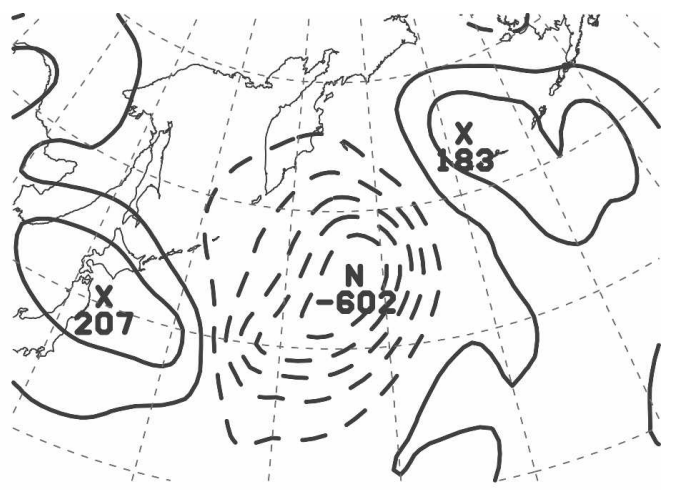

(b) DIAGNOSED NLB HEIGHT TEND

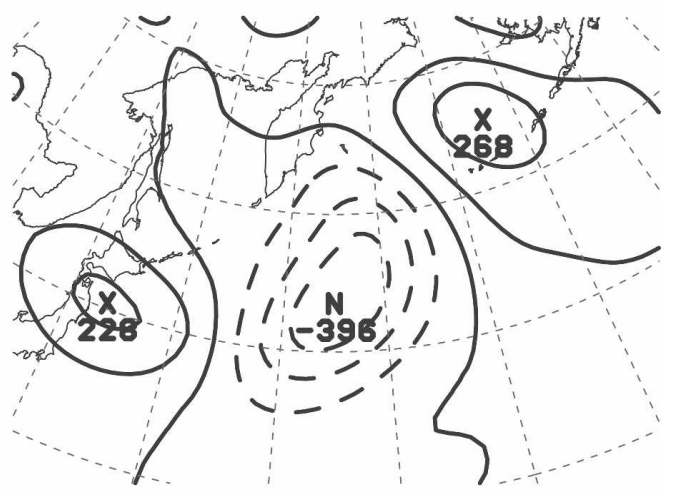

(d) ANALYZED NLB HEIGHT TEND

FIG. 5. Twelve-hour tendencies of upper-level heights (heights computed by inverting upper-level PV), centered at 0000 UTC 1 Apr 1987, at $300 \mathrm{hPa}$. (a), (c) Quasigeostrophic heights. (b), (d) Nonlinear balance heights. (a), (b) Diagnosed tendencies, computed by inverting PV advection. (c), (d) Analyzed tendencies, computed by subtracting

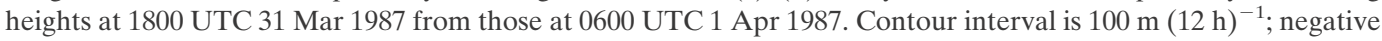
contours are dashed.

too the finite-difference upper-level height tendencies are similar (Figs. 5c,d). The diagnosed NLB height tendency (Fig. 5b) is verified against the finite-difference NLB height tendency (Fig. 5d), and the QG diagnosed height tendency (Fig. 5a) is verified against the finitedifference height tendency from QG inversion (Fig. 5c).

Despite being instantaneous, the diagnosed NLB height tendencies (Fig. 5b) underestimate the positive finite-difference tendencies (Fig. 5d) near the Bering Sea. A likely contributing factor to this difference is the neglect of diabatic generation of PV in the diagnosis. Latent heat release in the cyclone should reduce the upper-level PV over the region of ascent and act to cause heights to rise there and downstream within the ridge (e.g., Ahmadi-Givi et al. 2004), an effect only partially cancelled at upper levels by the diabatic PV generation below. Note, however, that only the height tendency associated with the instantaneous generation and destruction of $\mathrm{PV}$ is neglected. The ongoing effect of previously altered PV is fully included since the diagnosis is based upon the instantaneous PV distribution without regard for whether the PV had previously been conserved. Elsewhere, the two fields are in general agreement.

The agreement between the diagnosed and finitedifference QG height tendencies (Figs. 5a and 5c) is not as close. While the basic pattern is correct, there are differences in amplitude exceeding a factor of 2 in places, and many of the details in the diagnosed height tendency (such as those south of the jet) are absent in the analyzed height tendency. Since height falls are underestimated northeast of the system, the neglect of latent heat release appears to be having a similar detrimental effect in QG as in NLB.

Using the PTD framework, the upper-level PV advections inducing the height tendencies (and the height tendencies themselves, because both diagnostic systems are linear in the tendencies) can be subdivided into elements that are associated with specific dynamical processes (Nielsen-Gammon and Lefevre 1996). The formalism takes the same form in NLB and QG: differences arise in the mathematical form of the inversion 
operator and in the PV itself (pseudo-PV for QG, Ertel $\mathrm{PV}$ for NLB). We designate the upper-level PV as $q_{U}$ and the lower-level PV as $q_{L}$ and apply the subscripts $U$ and $L$ to all variables associated with the upper-level and lower-level PV, respectively. The upper and lower boundary conditions (here, potential temperature) are subsumed into the upper-level and lower-level PV. The heights associated with the upper-level PV, displayed in Figs. 4a,b,e,f, are

$$
\tilde{\Phi}_{U}^{(0)}=L^{-1}\left(\tilde{q}_{U}\right)
$$

in $\mathrm{QG}$ and

$$
\tilde{\Phi}_{U}=\mathcal{L}^{-1}\left(\tilde{Q}_{U}\right)
$$

in NLB. Here, the tilde represents the anomaly from the local time mean. An overbar will be used to represent the time mean itself. The $\mathcal{L}^{-1}$ in (18b) represents the inversion operation for solving (7)-(8).

The evolution of the upper heights in QG and NLB is given by

$$
\begin{gathered}
\frac{\partial \tilde{\Phi}_{U}^{(0)}}{\partial t}=L^{-1}\left(-\mathbf{V}^{(0)} \cdot \nabla q_{U}\right) \\
\frac{\partial \tilde{\Phi}_{U}}{\partial t}=\Lambda^{-1}\left(-\mathbf{V} \cdot \nabla Q_{U}\right) .
\end{gathered}
$$

The inversion operator in (19a) is identical to the one in (18a), but the nonlinear balance tendency inversion operator $\Lambda^{-1}$ incorporates solution of the five Eqs. (9)(13). Advection of the total upper-level PV, not merely the upper anomalies, must be included in the tendency diagnosis (19a)-(19b) since even advection of the timemean PV causes generation or evolution of PV anomalies and therefore height anomalies. In the NLB system, the advecting wind includes vertical motion (Davis et al. 1996). While the NLB forcing is specified only with the horizontal advection, the effect of vertical advection is included through the inversion operator.

The dynamically distinct elements of the advection term are as follows, here written in QG form:

$$
\begin{aligned}
\frac{\partial \Phi_{U}^{(0)}}{\partial t}= & L^{-1}\left(-\overline{\mathbf{V}}^{(0)} \cdot \nabla \bar{q}_{U}\right)+L^{-1}\left(-\tilde{\mathbf{V}}_{U}^{(0)} \cdot \nabla \bar{q}_{U}\right) \\
& +L^{-1}\left(-\tilde{\mathbf{V}}_{L}^{(0)} \cdot \nabla \bar{q}_{U}\right)+L^{-1}\left(-\overline{\mathbf{V}}^{(0)} \cdot \nabla \tilde{q}_{U}\right) \\
& +L^{-1}\left(\tilde{\mathbf{V}}^{(0)} \cdot \nabla \tilde{q}_{U}\right) .
\end{aligned}
$$

They are: advection of time-mean PV by the time-mean wind (expected to be small on the scale of baroclinic developments), advection of time-mean PV by the wind induced by upper-level PV anomalies (the Rossby wave propagation term; responsible for Rossby wave propa- gation and downstream development), advection of time-mean PV by the wind induced by lower-level PV anomalies (the baroclinic tendency term; responsible for baroclinic development), advection of upper-level PV anomalies by the time-mean winds (the deformation/advection term; responsible for barotropic and baroclinic deformation as well as downstream advection), and advection of upper-level PV anomalies by the winds induced by PV anomalies (the interaction term; generally affects the motion of PV anomalies more than their intensity). For a more complete discussion of the interpretation of all of these terms, see Nielsen-Gammon and Lefevre (1996) and Evans and Black (2003). Here we will concentrate on the baroclinic development, Rossby wave propagation, and time-mean advection/deformation terms.

The forcing in (9)-(13) includes not just the advection of potential vorticity by the nondivergent wind in (11) [expressed symbolically in (19b)] but also advection of potential temperature and absolute vorticity by the nondivergent wind in (12). These too are partitioned; for example, when the advection of upper-level PV anomalies by the time-mean wind is the forcing in (11), the forcing in (12) is the advection of the vorticity and potential temperature anomalies induced by upperlevel PV anomalies. The vorticity and potential temperature associated with the time-mean flow are not partitionable into upper and lower portions without introducing singularities into the omega equation forcing, so when the advection of upper or lower time-mean PV is a forcing in (11) the advection of time-mean vorticity and potential temperature is multiplied by 0.5 and used as a forcing in (12). This approach is one of several possible arbitrary partitionings that ensure that the full response is the sum of the partitioned responses.

Piecewise inversion of the prognostic balance system (9)-(13) is performed by (i) partitioning the wind, mass, and PV fields as in (20); (ii) applying 9-point smoothing to the wind and mass fields to improve convergence; (iii) computing advections from these partitioned fields; (iv) using the partitioned advections as the first guess for the three terms involving $\mathbf{v}_{\mathbf{h}}$ in (11) and (12); (v) solving (9)-(13) to obtain the irrotational and vertical winds and height/streamfunction tendencies; (vi) adding advections by these winds of the full (not partitioned) PV, potential temperature, and vorticity to the partitioned nondivergent advections in (11) and (12); (vii) solving (9)-(13) to obtain revised irrotational and vertical winds and height/streamfunction/PV tendencies; (viii) repeating steps (vi) and (vii) until the incremental updates are sufficiently small. Because of convergence problems, the last two terms on the right-hand side of (12) are not included in the inversion procedure. 

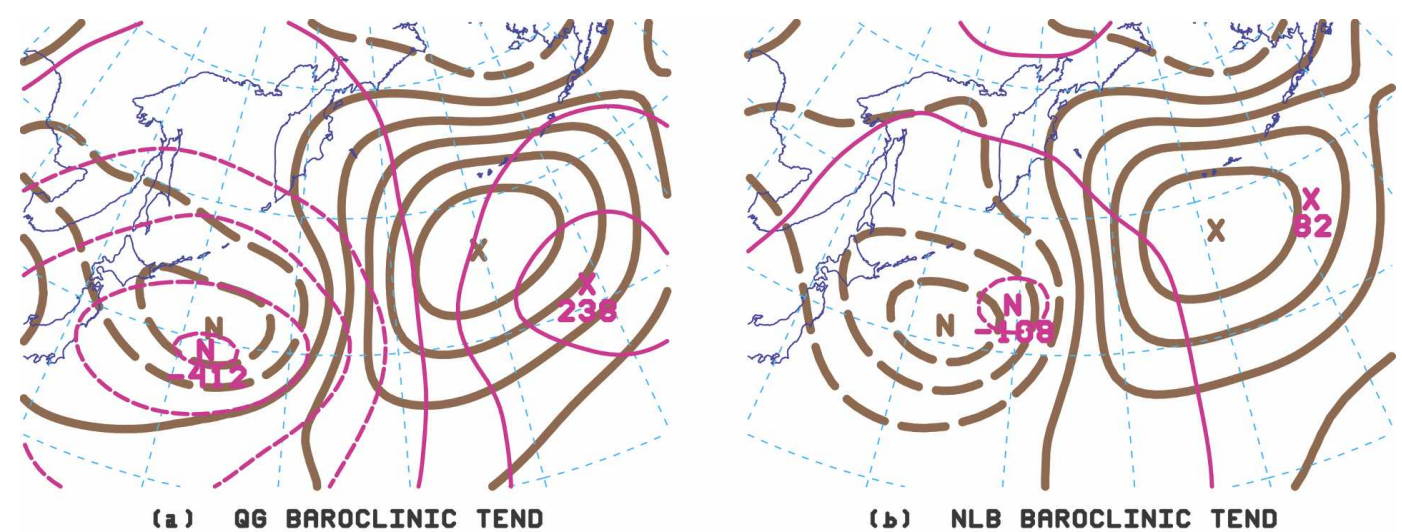

(b) NLB BAROCLINIC TEND

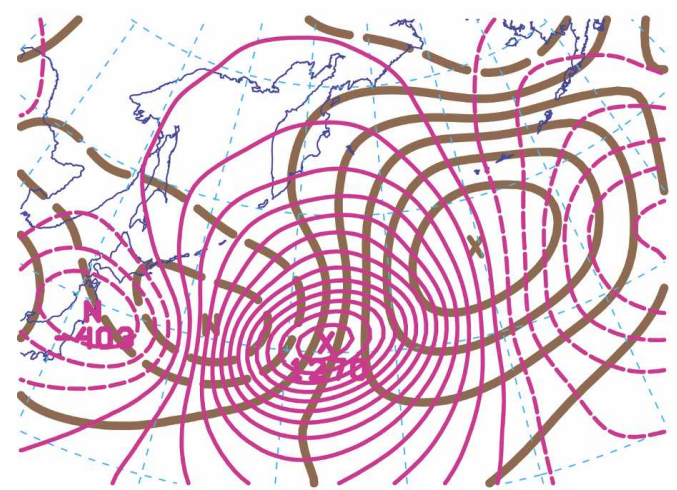

(c) QG RW PROP TEND

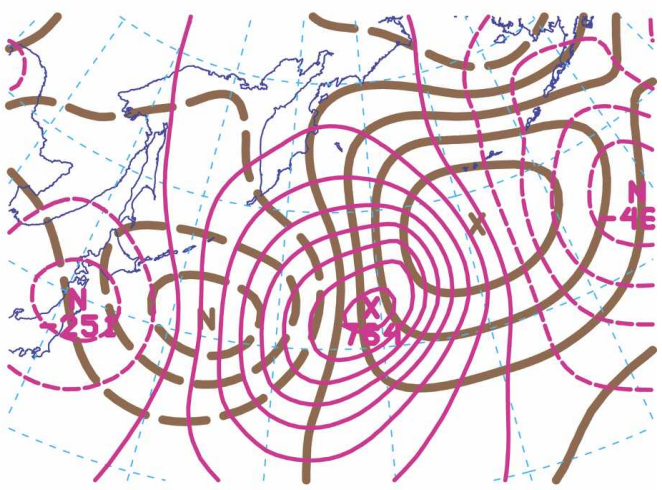

(d) NLB RW PROP TEND

FIG. 6. Diagnosed tendencies [(a), (c) quasigeostrophic; (b), (d) nonlinear balance] of 300-hPa geopotential height at 0000 UTC 1 Apr 1987 associated with the baroclinic tendency term [(a), (b) advection of upper-level time-mean PV by winds induced by lower-level PV anomalies] and the Rossby wave propagation term [(c), (d) advection of upper-level time-mean PV by winds induced by upper-level PV anomalies]. Tendencies are in magenta; contour interval is $100 \mathrm{~m}(12 \mathrm{~h})^{-1}$. The heights induced by upper-level PV anomalies are plotted in thick brown lines, with a 100-m contour interval. Negative contours are dashed.

The tendencies associated with the baroclinic development and Rossby wave propagation terms are shown in Fig. 6. The tendencies are overlaid on the heights induced by upper-level PV. The strength of a given cyclonic (anticyclonic) height anomaly is most simply measured by the minimum (maximum) perturbation height at its center. A nonzero tendency at the center of a height anomaly implies a change in intensity while a tendency dipole straddling a height anomaly implies advection or propagation. The baroclinic tendency term (Figs. 6a,b) is largely in phase with the height anomalies, meaning that the primary influence of lowlevel PV on the upper-level system is intensification. Such an effect is a direct consequence of a favorable (near $90^{\circ}$ ) phase shift between the heights induced by the upper-level and lower-level PV anomalies; this phase shift may be seen by comparing Fig. 4a with Fig. $4 c$ or comparing Fig. 4e with Fig. 4g.

The Rossby wave propagation tendencies (Figs. $6 c, d)$, on the other hand, are about a quarter wave- length out of phase from the upper-level height anomalies, indicating that the primary effect of winds induced by upper-level PV anomalies is to cause the height anomalies to move westward. This is the essence of Rossby wave propagation; as shall be seen shortly, the time-mean PV contours are oriented east-west (see also the full PV in Fig. 2).

Also significant is the fact that the tendencies are not precisely in phase or in quadrature. The baroclinic tendencies (Fig. 6a,b) are shifted downstream, indicating a tendency for the winds induced by low-level PV to enhance the eastward motion of the upper-level anomalies. This is the phase-locking mechanism discussed by Hoskins et al. (1985): because the lower-level anomalies are more than a quarter wavelength downstream of the upper-level anomalies, the baroclinic tendencies are acting in a manner so as to reduce the tilt. Meanwhile, the Rossby wave propagation tendencies (Fig. 6c,d) are positive in the core of the negative height anomaly (i.e., the trough). Figures $4 \mathrm{a}$ and $4 \mathrm{e}$ show that the trough is at 

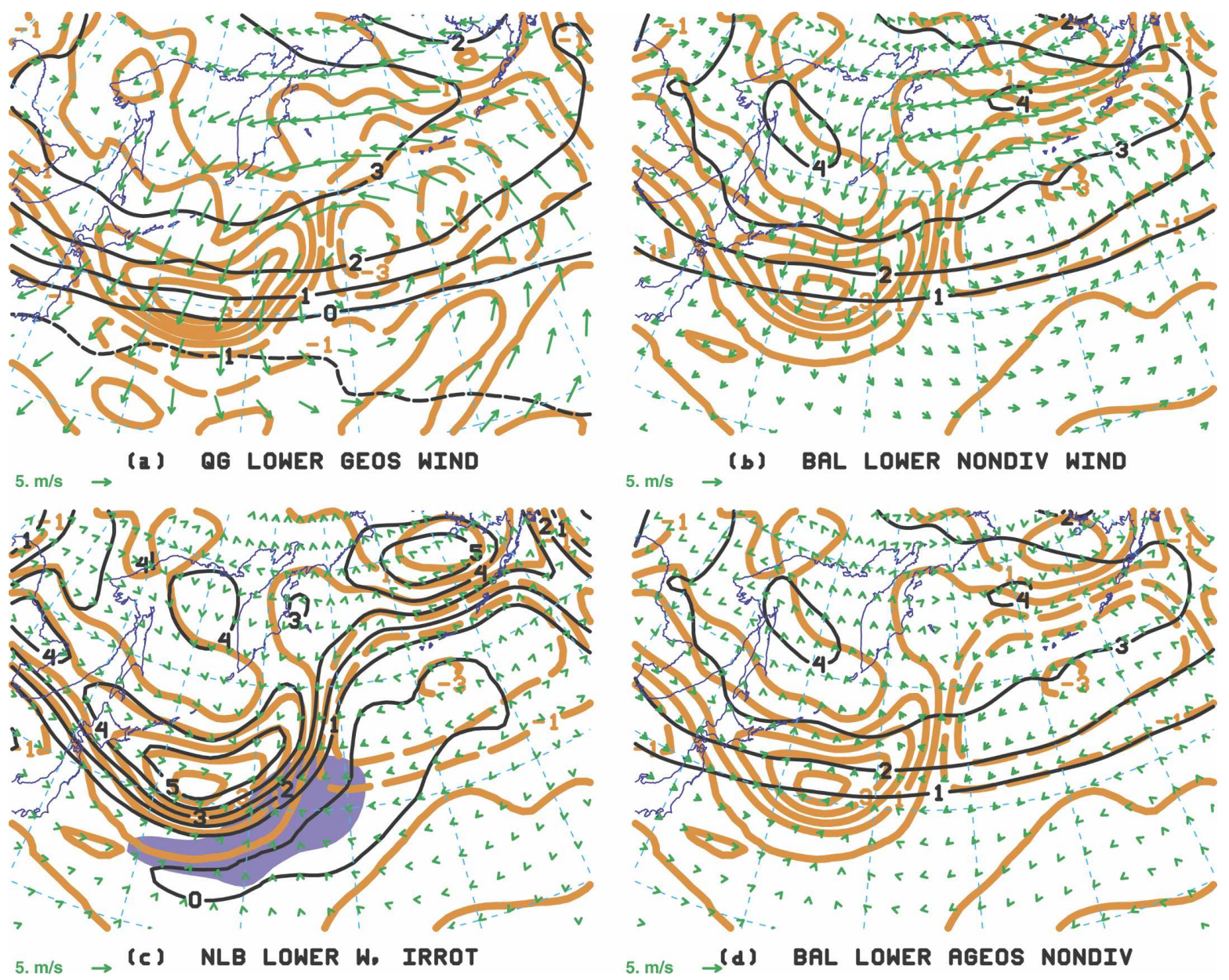

FIG. 7. Potential vorticity and winds associated with baroclinic development term at $300 \mathrm{hPa}$. (a) Time-mean pseudo-PV (black; contour interval $1 \times 10^{-4} \mathrm{~s}^{-1}$, negative values dashed), anomalous pseudo-PV (thick orange; contour interval $1 \times 10^{-4} \mathrm{~s}^{-1}$, negative values dashed), and winds induced by lower-level pseudo-PV anomalies (scale given in lower left). (b) As in (a) but for scaled Ertel PV and nondivergent winds induced by lower-level Ertel PV anomalies. (c) Full scaled Ertel PV (black; contour interval $1 \times 10^{-4} \mathrm{~s}^{-1}$ ), anomalous Ertel PV [orange, as in (b)], irrotational winds associated with baroclinic development term (scale given in lower left) and vertical velocities associated with baroclinic development term (shading interval is $0.5 \mathrm{~cm} \mathrm{~s}^{-1}$, positive values contoured, shading within $0.5 \mathrm{~cm} \mathrm{~s}^{-1}$ of zero suppressed). (d) As in (b) but with ageostrophic nondivergent winds induced by lower-level Ertel PV anomalies.

the western edge of a baroclinic wave packet, with much larger height anomalies to its east than to its west. According to previous studies of observed wave packets (e.g., Orlanski and Sheldon 1993; Chang 1999), such a trough should be weakening through Rossby wave propagation and downstream baroclinic development as the group velocity transports the Rossby wave energy eastward. The Rossby wave propagation tendencies confirm that this mechanism is operating here. Also, since the zero tendency line passes through the center of the downstream ridge in Fig. $6 c$,d, that ridge must be neither gaining or losing Rossby wave energy, consistent with its position closer to the center of the Rossby wave packet.

Comparing the QG and NLB tendencies, there are differences in both shape and amplitude. For both terms, the QG tendencies are larger, substantially so for the baroclinic tendencies (Fig. 6a). The locations of maxima and zero lines are generally similar, suggesting that, even though the QG tendencies are too strong, the diagnosed dynamical impacts of the various processes are qualitatively correct.

To determine the source of these differences and to illustrate the dynamical processes behind the piecewise height tendencies, we now examine the wind and PV fields associated with the advections themselves. The baroclinic term is broken down in Fig. 7, and the Rossby wave term in Fig. 8. In both figures, the timemean PV is shown in black contours. The time-mean PV and advecting winds are overlaid onto the upper- 

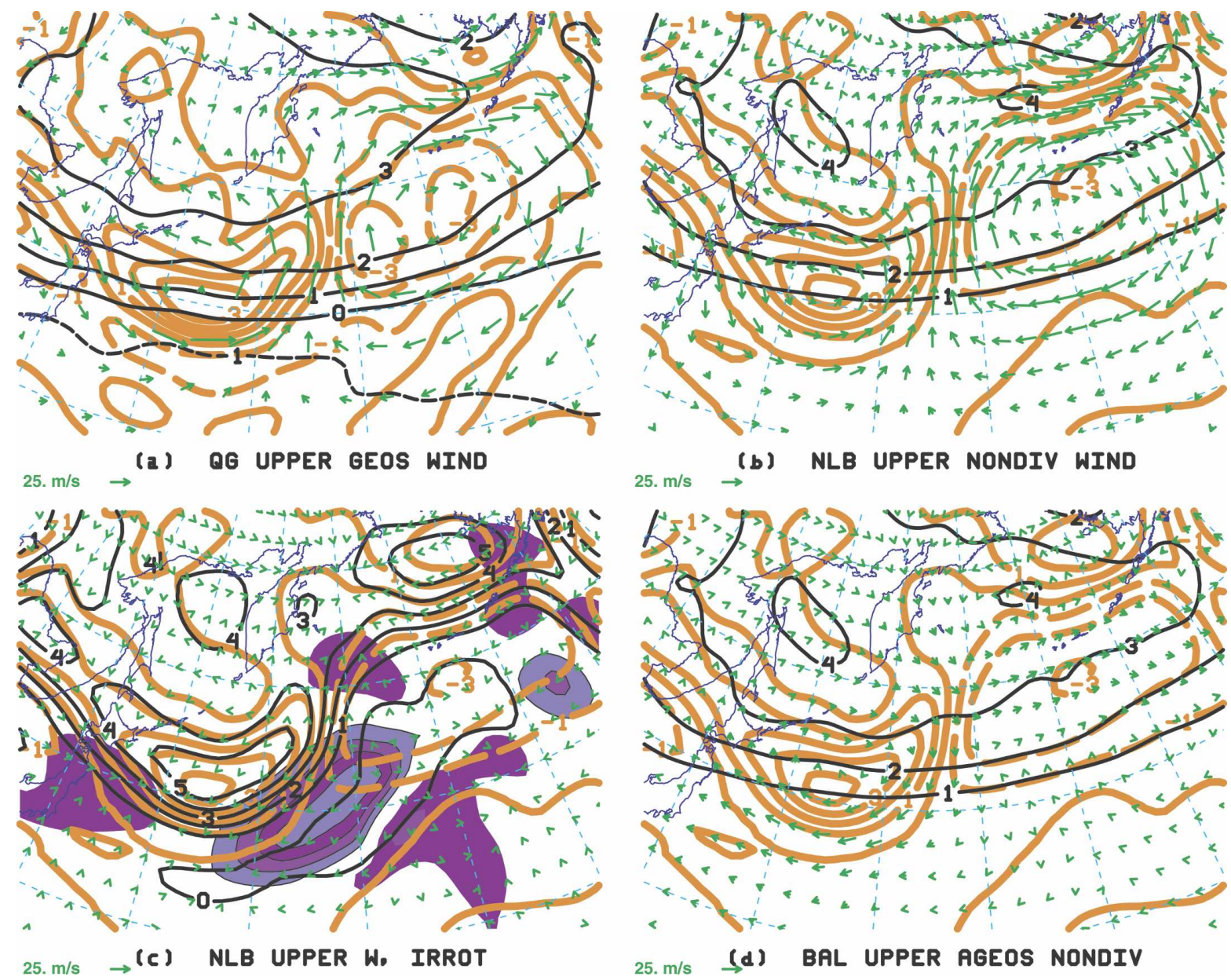

FIG. 8. As in Fig. 7 but for PV and winds associated with Rossby wave propagation term. Winds and vertical velocities are induced by upper-level pseudo-PV or Ertel PV anomalies.

level PV anomalies, shown in thick gray contours. Note that Figs. 7a, 8a show the pseudo-PV while the other panels show the Ertel PV, scaled by (14).

Figures $7 \mathrm{a}$ and $7 \mathrm{~b}$ display the classical pattern of baroclinic amplification. The winds induced by the lower-level PV are predominantly cyclonic, associated with the frontal thermal wave and any lower-level PV anomalies produced by latent heat release. To the west, these winds are blowing across the time-mean PV contours, acting to advect high values of PV southward. In isolation, such advection would generate a positive PV anomaly. Since there is already a positive PV anomaly there, the advection will act to intensify the existing anomaly. To the east, the winds are advecting low PV northward, thereby intensifying the negative PV anomaly that is already present there. Although not shown here, the winds induced by the upper-level PV are similarly acting to amplify the existing frontal wave and surface potential temperature anomaly couplet.
We have already noted that the resulting upper-level height tendencies are not quite in phase with the heights themselves, implying that the winds associated with lower-level PV are altering the tilt of the system as well as amplifying the upper-level wave. In the words of Hoskins et al. (1985), "The induced velocity field of each Rossby wave keeps the other in step, and makes the other grow." Fig. 7 is a qualitative depiction of this process in action in the real atmosphere, and the calculations shown in Fig. 6 quantify the effect.

The differences in the baroclinic tendencies between QG and NLB appear to be due largely to the differences in the nondivergent (or geostrophic) winds induced by the lower-level PV. As seen in Figs. 4c and 4g, the induced heights (and consequently the induced geostrophic winds as well) penetrate much more strongly to upper levels from lower levels in the QG framework. As a result, the winds induced at $300 \mathrm{hPa}$ are more than twice as strong in Fig. 7a as in Fig. 7b. Also contributing 
to the difference in tendencies is a stronger time-mean PV gradient (and larger total north-south time-mean PV difference) in the QG framework compared to the Ertel framework.

One might wonder whether the overestimate of nondivergent wind speeds by QG in cyclonic flow may also contribute to the height tendency difference. Figure 7d shows that the horizontal scale of cyclonic flow is so large that the ageostrophic component of the nondivergent wind is negligible.

The description of baroclinic amplification by Hoskins et al. (1985) considered only the horizontal nondivergent wind, but the balanced height tendencies are a consequence of the full balanced advection, including the horizontal nondivergent wind, the horizontal irrotational wind, and the vertical motion, with the latter two diagnosed from the prognostic balance equations (Davis and Emanuel 1991).

The irrotational horizontal wind (Fig. 7c) does not play a significant role in baroclinic amplification in this case, and that result appears to be general. Because the upward and downward motion will be strongest beneath the upper-level jet stream, the upper-level convergence and divergence will act primarily to spread out or contract the upper-level PV contours rather than altering the strength of particular anomalies.

The NLB vertical motion pattern (Fig. 7c) is dominated by descent in the trough. Because descent at this level serves to bring high-PV air downward into the troposphere, the descent is acting to amplify the trough. Thus, vertical motion is contributing favorably to the baroclinic amplification height tendencies. The conventional QG system does not include this vertical advection but, as discussed by Nielsen-Gammon and Gold (2008), a QG potential vorticity can be defined that responds to both vertical and horizontal advection.

The pattern of vertical motion in Fig. 7c is explained by isentropic upglide. Since the baroclinic amplification term isolates anomalous low-level winds in a time-mean environment, this specific component of vertical motion is caused from an isentropic point of view by the north-south motion of air along isentropic surfaces that are sloping because of the presence of time-mean baroclinicity (Fig. 2a). The same process, now associated with winds induced by upper-level PV anomalies, produces the vertical motion pattern in Fig. 8c below. In contrast, the vertical motion associated with time-mean winds advecting PV anomalies (Fig. 9c, below) would be associated with the "vacuum cleaner effect" (Hoskins et al. 1985) of bulging isentropic surfaces present in a shear flow. Thus, Bal-PTD produces a vertical motion decomposition analogous to that discussed by Dixon et al. (2003) in the QG framework.
There is much better agreement between the geostrophic (Fig. 8a) and nondivergent (Fig. 8b) winds induced by upper-level PV anomalies than those induced by lower-level anomalies (Figs. 7a-b). Consequently, the associated height tendencies (Figs. 6c-d) are also in better agreement. Northward advection of low-PV air is taking place between the trough and the downstream ridge, leading to positive height tendencies there. This tendency, acting alone, would cause the negative height anomaly (the ridge) to propagate westward. Likewise, southward advection upstream of the trough leads to negative height tendencies and westward propagation of the trough.

Close examination of Figs. 8a-b reveals that in the center of the positive PV anomaly that constitutes the trough east of Japan, the wind induced by the upperlevel PV anomalies is weakly southerly, acting to advect low-PV air northward. By comparison with Fig. 2c-d, these advections are acting to weaken the trough, in addition to their propagation role. The reason such a wind exists may be explained as follows (NielsenGammon 1995): In a pure Rossby wave train, the wind in the center of the trough is zero. The trough itself induces a cyclonic circulation that is centered over the trough and thus does not contribute to advection in the center of the trough itself. The upstream ridge would induce northerly flow downstream, in the trough, while the downstream ridge would induce southerly flow upstream, in the trough. The effects of the two ridges cancel each other, leaving zero wind in the center of the trough. Similar cancellation takes place between more distant wave elements. However, when the wave is asymmetric, cancellation does not occur. In this case, there is a strong downstream ridge but no corresponding upstream ridge. Thus, the southerlies induced by the downstream ridge are not mitigated by an upstream ridge, and net weakening of the trough results.

The ageostrophic nondivergent winds (Fig. 8d) weaken the advection just downstream of the trough where the flow curvature is cyclonic and the wind subgeostrophic and enhance the advection just downstream of the ridge where the flow curvature is anticyclonic and the wind supergeostrophic. The influence of the ageostrophic winds appears to be small. As before, the vertical motion (Fig. 8c) is in phase with the PV advection, amplifying its effect.

The other important term in the piecewise height tendencies at upper levels is due to advection of upperlevel PV anomalies by the time-mean flow. This term is here called the time-mean advection/deformation term. The comparison of QG and NLB diagnosis for this term is shown in Fig. 9. As would be expected, differences 

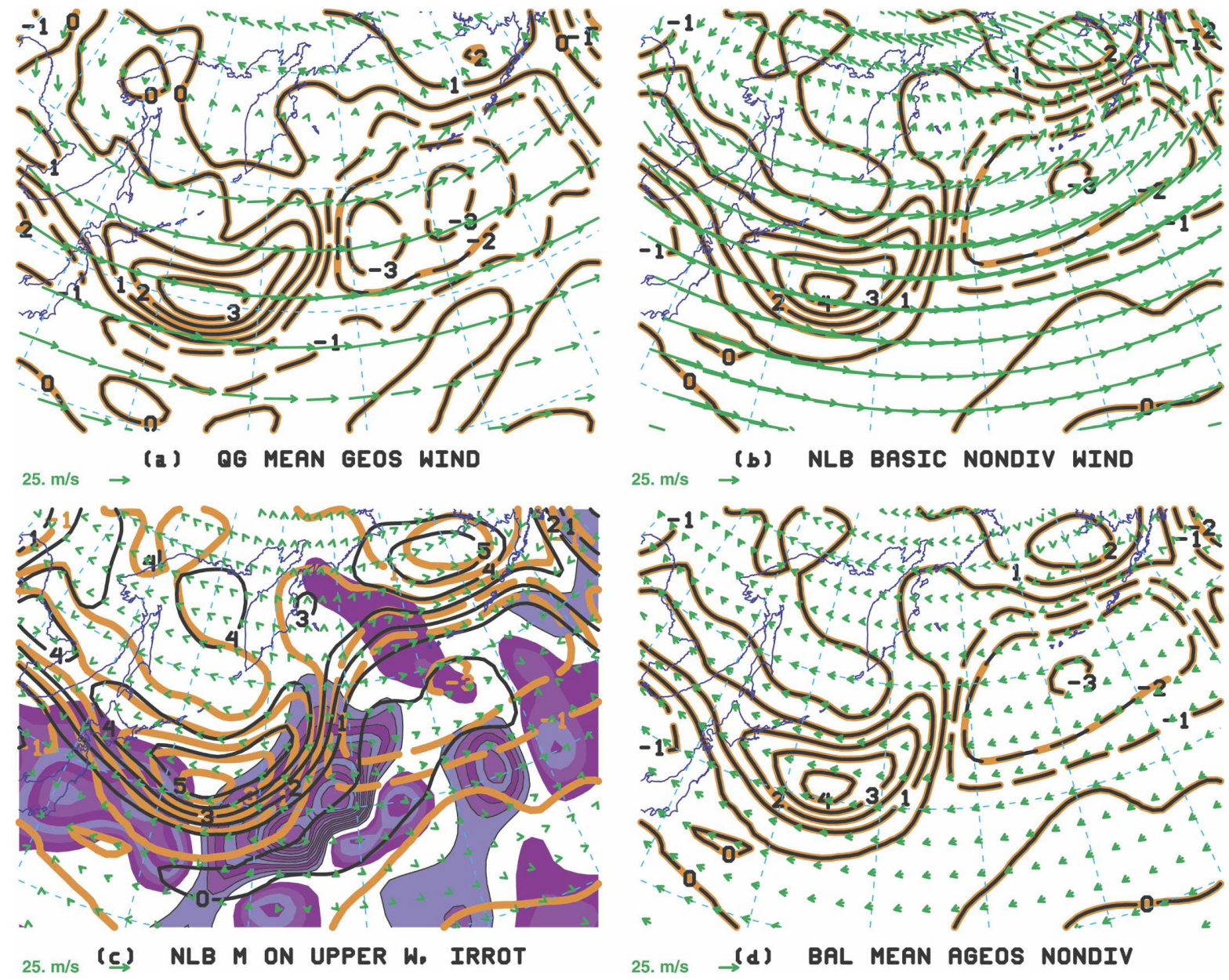

FIG. 9. As in Fig. 7 but for PV and winds associated with the time-mean advection/deformation term. Winds and vertical velocities are associated with time-mean flow interacting with upper-level PV anomalies.

between the geostrophic and nondivergent time-mean winds are small (Figs. 9a-b), and the nondivergent ageostrophic wind (Fig. 9d) is present largely because the jet stream curves cyclonically as it circumnavigates the globe. As seen in Fig. 9c, this term is associated with the largest magnitudes of vertical motion of all the terms considered here.

Figures $10 \mathrm{a}-\mathrm{b}$ provide the height tendencies associated with the advections shown in Fig. 9. As with the Rossby wave propagation term (Figs. 6c-d), the QG tendencies are considerably larger than the NLB tendencies. While stronger QG winds are responsible for the difference in the Rossby wave term (cf. Figs. 8a-b), the difference in the advection/deformation term is largely due to the effect of vertical motion in the NLB system. The strong upward motion ahead of the trough produces negative vertical PV advection, partially cancelling the horizontal advection of the PV anomaly.

While the dominant effect of the advection/defor- mation term is downstream translation of the height anomalies, intensification or weakening can arise in this term through the effects of horizontal deformation or vertical shear rearranging the PV anomalies. This effect can sometimes be significant (Nielsen-Gammon and Lefevre 1996), but in this instance the tendencies at the center of the trough and downstream ridge are near zero.

The tendencies associated with the remaining terms are shown in Fig. 10c-d. Again the tendencies at the center of the height anomalies are small. The tendency pattern is largely produced by the advection of upperlevel anomalies by winds associated with upper-level anomalies, which acts to transport both anomalies northward. Unlike Fig. 10c, Fig. 10d includes the effect of low-level processes through the omega equation and the resulting upper-level advection of PV by the vertical circulation. The net effect of these processes on the intensity of the upper-level height anomalies is small. 

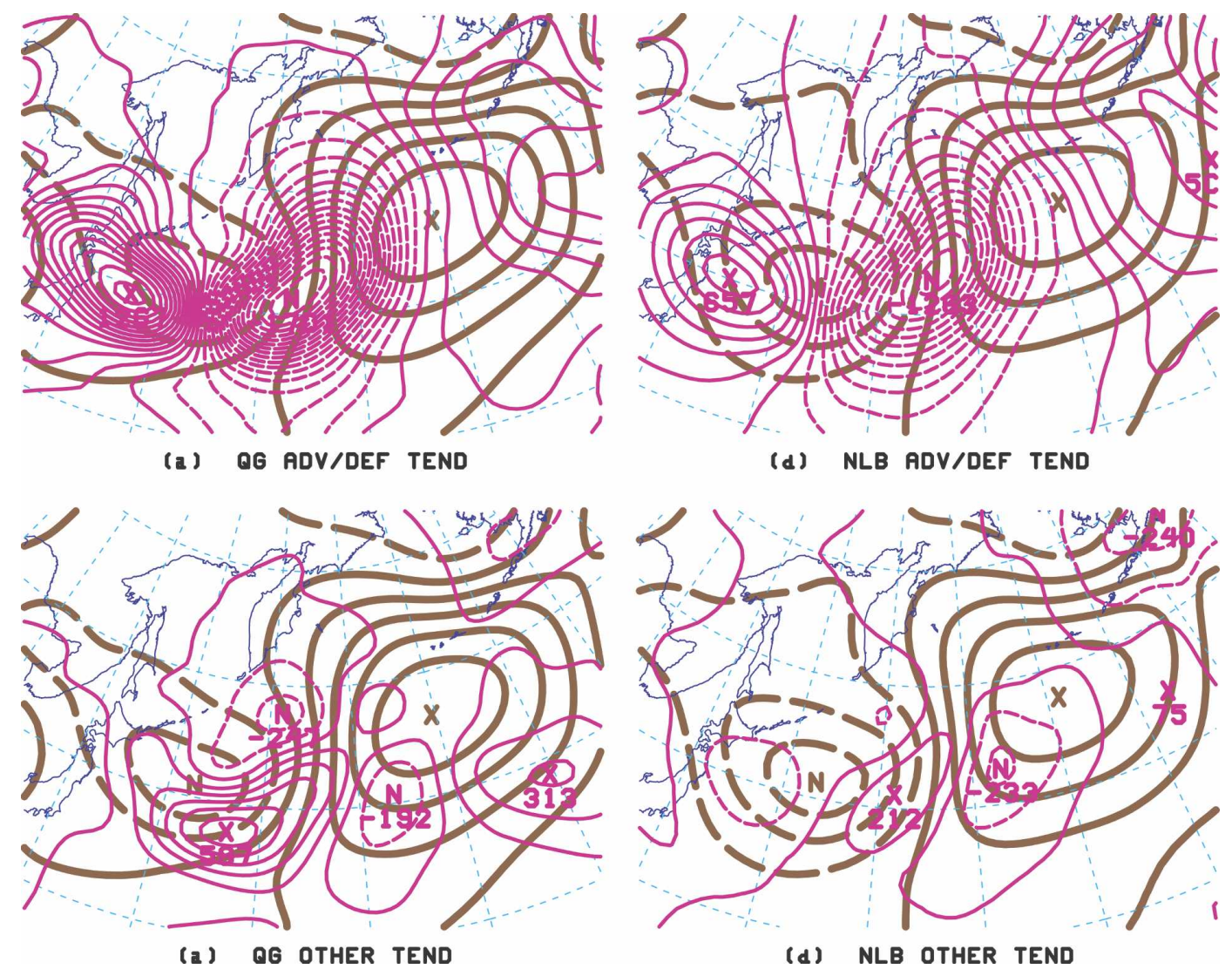

FIG. 10. Tendencies and anomalous heights, as in Fig. 6, but for (a), (b) the upper-level time-mean advection/deformation term and (c), (d) the remaining terms.

\section{Concluding remarks}

The nonlinear balance system permits not just quantitative diagnosis of the influence of PV anomalies on the static atmosphere, but also their influence on the dynamical evolution of the atmosphere. The use of piecewise tendency diagnosis with the nonlinear balance system is here called Bal-PTD. Both Bal-PTD and QG PTD have been applied to a rapidly intensifying oceanic cyclone, and the origin and nature of quantitative differences in diagnoses using the two systems have been explored.

The differences between QG and NLB diagnosis of this event are caused, in order of importance, by differences in the upward and downward influence of PV anomalies, differences in the magnitude of the timemean PV gradients, and differences in the treatment of vertical motion. Despite these differences, the overall patterns and phase relationships among the different tendency terms were generally consistent between QG and NLB, meaning that individual processes can be diagnosed to at least qualitative accuracy with either system. However, because of the differences in vertical penetration, the diagnosis of relative importance of different dynamical mechanisms may suffer when the less accurate QG system is used. When considering the use of PTD, this shortcoming must be weighted against the relative ease of implementation of QG inversion.

One result that appears to be general is that QGPTD overestimates the effect of lower-level PV (and surface $\theta$ ) in amplifying and controlling the motion of the upper-level system. One would expect that QG and NLB would be more similar in events with smaller Ro and less similar in events with larger Ro. In circumstances such as tropical cyclones and other large mesoscale disturbances, NLB can succeed where QG fails.

While treated separately here, the difference in the magnitude of the time-mean PV gradients is at least partly a consequence of the difference in the treatment of vertical motion. As shown by Charney and Stern (1962), the advection of pseudo-PV on horizontal surfaces is approximately proportional to the advection of Ertel PV on isentropic surfaces. If the vertical motion is in phase with the horizontal advection, as seen above, the total advection of Ertel PV will be stronger than the horizontal advection of Ertel PV. To produce the same 
advection with just the horizontal winds, the pseudoPV must have a stronger horizontal gradient than the Ertel PV, and this comes about through the addition of the temperature anomaly term (Nielsen-Gammon and Gold 2008). Thus, the opposing effects of vertical motion and time-mean PV gradient differences are partly cancellation by design, leaving the vertical penetration differences alone as the main cause of differences in diagnosis between QG and NLB.

Further investigation is required to determine the nature of differences in low-level diagnosis. Also unknown is whether the use of a different slaving variable, such as vorticity, would improve the performance of QG PTD and reduce the differences between QG and NLB (Nielsen-Gammon and Gold 2008). The work of Thorpe and Bishop (1995) suggests that the differences between penetration depths in QG and NLB are inherent in the QG approximation, so the primary diagnostic differences between QG and NLB may be independent of a slaving variable.

Also, the comparisons performed here were only for a single time. If one intends to track the evolution of the system dynamics through PV, a dynamical framework using Ertel PV has the advantage that Ertel PV will be exactly conserved in dry inviscid dynamics. Nonconservation of Ertel PV may directly be attributed to diabatic and frictional processes, whereas the nonconservation of pseudo-PV, linearized PV, or any other approximated $\mathrm{PV}$, is ambiguous.

In summary, QG diagnosis was adequate to determine the signs and overall spatial patterns of the various forcing terms important to this case of rapid midlatitude cyclogenesis. However, for quantitative partitioning of the relative importance of the various dynamical processes, the use of Bal-PTD is necessary.

Acknowledgments. Christopher Davis and an anonymous reviewer helped greatly to improve the clarity of an earlier version of this manuscript. This work was partially supported by the National Science Foundation through Grants ATM-9521383 and ATM-0089906.

\section{REFERENCES}

Ahmadi-Givi, F., G. C. Craig, and R. S. Plant, 2004: The dynamics of a midlatitude cyclone with very strong latent heat release. Quart. J. Roy. Meteor. Soc., 130, 295-323.

Bishop, C. H., and A. J. Thorpe, 1994: Potential vorticity and the electrostatics analogy: Quasigeostrophic theory. Quart. J. Roy. Meteor. Soc., 120, 713-731.

Bresky, W. C., and S. J. Colucci, 1996: A forecast and analyzed cyclogenesis event diagnosed with potential vorticity. Mon. Wea. Rev., 124, 2227-2244.

Chang, E. K. M., 1999: Characteristics of wave packets in the upper troposphere. Part I: Northern Hemisphere winter. $J$. Atmos. Sci., 56, 1708-1728.

Charney, J. G., 1962: Integration of the primitive and balance equations. Proc. Symp. Numerical Weather Prediction, Tokyo, Japan, Japan Meteorological Agency, 131-152.

— jets in a rotating atmosphere. J. Atmos. Sci., 19, 159-172.

Davis, C. A., 1992: Piecewise potential vorticity inversion. J. Atmos. Sci., 49, 1397-1411.

- and K. A. Emanuel, 1991: Potential vorticity diagnostics of cyclogenesis. Mon. Wea. Rev., 119, 1929-1953.

, E. D. Grell, and M. A. Shapiro, 1996: The balanced dynamical nature of a rapidly intensifying oceanic cyclone. Mon. Wea. Rev., 124, 3-26.

Dixon, M. A. G., A. J. Thorpe, and K. A. Browning, 2003: Layerwise attribution of vertical motion and the influence of potential-vorticity anomalies on synoptic development. Quart. J. Roy. Meteor. Soc., 129, 1761-1778.

Evans, K. J., and R. X. Black, 2003: Piecewise tendency diagnosis of weather regime transitions. J. Atmos. Sci., 60, 1941-1959.

Gold, D. A., 1996: A quantitative analysis of baroclinic instability in extratropical cyclogenesis. M.S. thesis, Dept. of Atmospheric Sciences, Texas A\&M University, 102 pp.

Grotjahn, R., 1996: Vorticity equation terms for extratropical cyclones. Mon. Wea. Rev., 124, 2843-2858.

Hoskins, B. J., M. E. McIntyre, and A. Robertson, 1985: On the use and significance of isentropic potential vorticity maps. Quart. J. Roy. Meteor. Soc., 111, 887-946.

Kalnay, E., and Coauthors, 1996: The NCEP/NCAR 40-Year Reanalysis Project. Bull. Amer. Meteor. Soc., 77, 437-471.

McWilliams, J. C., and P. R. Gent, 1980: Intermediate models of planetary circulations in the atmosphere and ocean. J. Atmos. Sci., 37, 1657-1678.

Nielsen-Gammon, J. W., 1995: Dynamical conceptual models of upper-level mobile trough formation: Comparison and application. Tellus, 47A, 705-721.

- and R. J. Lefevre, 1996: Piecewise tendency diagnosis of dynamical processes governing the development of an uppertropospheric mobile trough. J. Atmos. Sci., 53, 3120-3142.

- and D. A. Gold, 2008: Dynamical diagnosis: A comparison of quasigeostrophic and Ertel potential vorticity. Sanders Symposium Monograph, Meteor. Monogr., No. 55, Amer. Meteor. Soc., in press.

Orlanski, I., and J. Sheldon, 1993: A case of downstream baroclinic development over western North America. Mon. Wea. Rev., 121, 2929-2950.

Pauley, P. M., and S. J. Nieman, 1992: A comparison of quasigeostrophic and nonquasigeostrophic vertical motions for a model-simulated rapidly intensifying marine extratropical cyclone. Mon. Wea. Rev., 120, 1108-1134.

Rotunno, R., and J.-W. Bao, 1996: A case study of cyclogenesis using a model hierarchy. Mon. Wea. Rev., 124, 1051-1066.

Simmons, A. J., and B. J. Hoskins, 1976: Baroclinic instability on the sphere: Normal modes of the primitive and quasigeostrophic equations. J. Atmos. Sci., 33, 1454-1477.

Thorpe, A. J., and C. H. Bishop, 1995: Potential vorticity and the electrostatics analogy: Ertel-Rossby formulation. Quart. J. Roy. Meteor. Soc., 121, 1477-1495.

Vasilj, J. M., and P. J. Smith, 1997: A comparison of extended and quasigeostrophic dynamics for a case of small-Rossby number extratropical cyclone development. Mon. Wea. Rev., 125, 3347-3356. 